\title{
Rivaroxaban Treatment for Heparin-Induced Thrombocytopenia: A Case Report and a Review of the Current Experience
}

\author{
Mohamed Aon (iD) ${ }^{1,2}$ and Omar Al-Shammari ${ }^{1}$ \\ ${ }^{1}$ Department of Medicine, Jahra Hospital, Ministry of Health (HOM), Jahra city, Kuwait \\ ${ }^{2}$ Department of Medicine, Faculty of Medicine, Cairo University, Giza, Egypt \\ Correspondence should be addressed to Mohamed Aon; maon2004@cu.edu.eg
}

Received 30 June 2020; Revised 14 August 2020; Accepted 26 August 2020; Published 4 September 2020

Academic Editor: Gergely Feher

Copyright ( 2020 Mohamed Aon and Omar Al-Shammari. This is an open access article distributed under the Creative Commons Attribution License, which permits unrestricted use, distribution, and reproduction in any medium, provided the original work is properly cited.

\begin{abstract}
Heparin-induced thrombocytopenia is a life-threatening complication of exposure to heparin. Heparin-induced thrombocytopenia results from an autoantibody directed against platelet factor 4 in complex with heparin. Heparin-induced thrombocytopenia is traditionally treated with bivalirudin, argatroban, danaparoid, or fondaparinux. Recently, direct oral anticoagulants administration to treat heparin-induced thrombocytopenia has been reported. Direct oral anticoagulants do not cause platelet activation in the presence of heparin-platelet factor 4 antibodies, nor do they provoke autoantibody production. Direct oral anticoagulants offer advantages such as consistent and predictable anticoagulation, oral administration with good patient compliance, and a good safety profile. We report a case of heparin-induced thrombocytopenia with deep venous thrombosis successfully treated with rivaroxaban and review the current experience with rivaroxaban for the treatment of heparininduced thrombocytopenia.
\end{abstract}

\section{Introduction}

Heparin-induced thrombocytopenia (HIT) is a clinical syndrome complicating exposure to heparin that occurs in a small percentage of patients. HIT is caused by antibodies to complexes of platelet factor 4 (PF4) and heparin that can cause thrombosis along with thrombocytopenia. HIT diagnosis rests on the presence of thrombocytopenia and/or thrombosis after heparin exposure while excluding other possible causes of thrombocytopenia [1]. HIT requires immediate treatment to reduce the risk of serious thrombosis. There are several nonheparin anticoagulants that can be used in the treatment of HIT, e.g., argatroban, bivalirudin, danaparoid, and fondaparinux. Accumulating evidence suggests that direct oral anticoagulants (DOACs) are effective in treating HIT [2].

In this article, we report a case of HIT complicated with deep venous thrombosis (DVT) successfully treated with rivaroxaban and review the accumulating experience with rivaroxaban for the treatment of HIT.

\section{Case Presentation}

A 25-years-old male presented to Jahra Hospital, Kuwait, with confusion after the ingestion of methanol. In the emergency room, he had high anion gap metabolic acidosis ( $\mathrm{pH} 6.8, \mathrm{HCO}_{3} 8 \mathrm{mmol} / \mathrm{L}$, anion gap $31 \mathrm{mmol} / \mathrm{L}$ ), elevated serum lactate $(16.6 \mathrm{mmol} / \mathrm{L})$, and hypoglycemia. Treatment was started with intravenous dextrose, thiamine, and sodium bicarbonate infusion. Due to persistent acidosis, dialysis was started through a right femoral catheter. After dialysis, his metabolic parameters improved, and the catheter was removed. His medication included enoxaparin $40 \mathrm{mg}$ once daily for DVT prophylaxis, omeprazole, folic acid, and thiamine. On the 5th day of hospital stay, the patient's platelet count decreased (Figure 1), with normal white blood cells, hemoglobin, and coagulation profile, so his enoxaparin prophylaxis was substituted with fondaparinux $2.5 \mathrm{mg}$ once daily. On the 6th day of hospital stay, he started to develop right-sided thigh pain and swelling. Urgent ultrasound examination of the deep venous system revealed right 


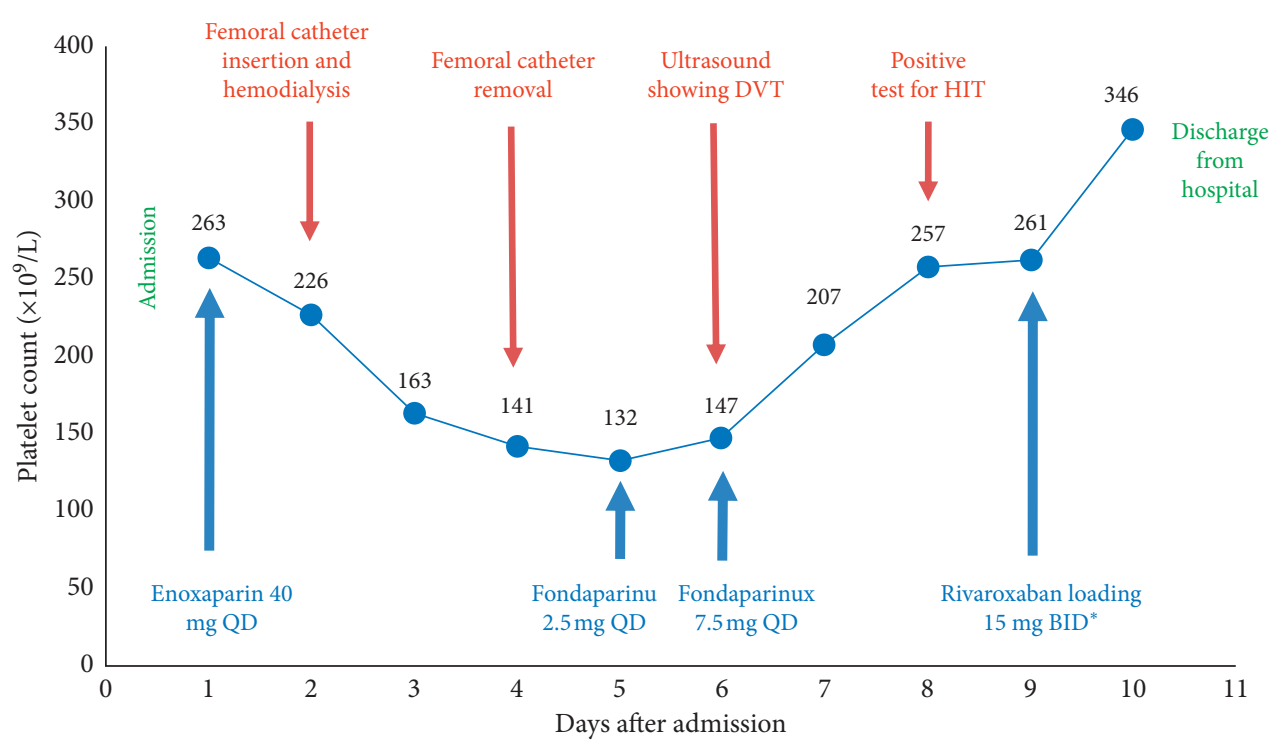

FIGURE 1: Platelet count charted against time and timeline of clinical events and medications used until day of discharge. QD, once daily; BID, twice per day; DVT, deep venous thrombosis; HIT, heparin-induced thrombocytopenia. *Loading for 21 days and then 20 mg QD to complete a total of three months.

external iliac, femoral, and popliteal vein recent thrombosis. Fondaparinux was increased to a therapeutic dose according to his body weight ( $7.5 \mathrm{mg}$ once daily) and HIT serology was sent. Over the next days, the patient's condition was improving, and blood examination showed recovery of platelet count (Figure 1). Heparin-induced anti-platelet antibodies were detected at 0.846 optical density (OD) units, and the confirmatory functional assay was positive. Given his clinical and laboratory improvement, we decided to start him on rivaroxaban. Loading dose $15 \mathrm{mg}$ twice daily for 21 days was started and the patient was released to outpatient care. Follow-up in outpatient revealed continued clinical improvement. The treatment was well tolerated with no adverse effects and the dosing was switched to $20 \mathrm{mg}$ once daily to complete a total of three months of treatment. Three months after the hospital discharge, the patient underwent outpatient examination that revealed a good overall condition with a normal platelet count and recanalization of the deep venous system on ultrasound examination. Rivaroxaban was stopped and the patient was instructed to avoid heparin for life.

\section{Discussion}

HIT is a clinical syndrome caused by antibodies to complexes of PF4 and heparin. This syndrome has also been called heparin-induced thrombocytopenia and thrombosis (HITT) because antibodies can cause thrombosis along with thrombocytopenia [1]. Antibody-coated platelets are removed by the reticuloendothelial system causing thrombocytopenia. Consumption of platelets at sites of thrombosis is another mechanism of thrombocytopenia in HIT [3]. HIT is a prothrombotic disorder and the primary mechanism of thrombosis is due to the binding of HIT antibodies to the platelet which elicits platelet activation and release of procoagulant microparticles. Platelet activation in HIT is also accompanied by endothelial cell activation and injury leading to increased tissue factor expression and thrombin generation [4]. Affected individuals have a $50 \%$ risk of developing new thromboembolic events which can involve both the arterial and the venous systems. The mortality rate is approximately $15 \%$, and $10 \%$ of patients require amputations or suffer another major morbidity [5]. HIT is more common in surgical patients compared with medical patients and more common among females. Absolute risks of developing HIT after exposure to prophylactic unfractionated heparin is $2.6 \%$ versus $0.2 \%$ after low molecular weight heparin (LMWH) exposure [6]. HIT diagnosis rests on the presence of thrombocytopenia and/or thrombosis in temporal association with heparin exposure while excluding other causes of thrombocytopenia. If HIT is suspected, it is recommended to use the 4Ts score to estimate the probability of HIT [7]. If there is an intermediate to high probability of HIT (4Ts score $\geq 4$ ), heparin should be discontinued and an immunoassay for HIT antibodies is obtained. If the immunoassay is positive, a functional assay is used to confirm the results. A functional assay may not be necessary for patients with a high probability score and a strongly positive immunoassay $>2.0$ OD units [8].

Given that platelet drop is $50 \%$ on the 5 th day after LMWH exposure, associated with a new DVT, and no other causes for thrombocytopenia are present, our patient's 4Ts score is 7 points, i.e., a high probability of HIT. His immunoassay is positive (OD 0.846 ; OD values $\geq 0.400$ are considered positive in our reference laboratory and the confirmatory test is positive).

Once HIT is diagnosed, heparin must be discontinued, and a nonheparin anticoagulant is used instead. The latest American Society of Hematology (ASH) HIT treatment guidelines endorse the use of either argatroban, bivalirudin, danaparoid, or fondaparinux. Because of their short 
TABLE 1: Cohort studies and case reports of rivaroxaban for the treatment of HIT.

\begin{tabular}{|c|c|c|c|c|c|c|}
\hline $\begin{array}{l}\text { Study } \\
\text { reference }\end{array}$ & $\begin{array}{l}\text { Number of } \\
\text { patients }\end{array}$ & HITT* & $\begin{array}{l}\text { Rivaroxaban treatment for } \\
\text { acute or subacute HIT* }\end{array}$ & $\begin{array}{l}\text { Primary or } \\
\text { secondary } \\
\text { treatment }\end{array}$ & $\begin{array}{l}\text { Extension of thrombosis } \\
\text { or major bleeding }\end{array}$ & Dose* \\
\hline$[2,9]$ & 28 & $12 / 28$ & $\begin{array}{l}19 / 28 \text { acute } \\
9 / 28 \text { subacute }\end{array}$ & $\begin{array}{l}13 / 28 \text { primary } \\
15 / 28 \text { secondary }\end{array}$ & $\mathrm{Yes}^{\mathrm{a}, \mathrm{b}}$ & $\begin{array}{l}\text { 20/28 standard } \\
\text { dose } \\
4 / 2820 \mathrm{mg} \text { once } \\
\text { daily } \\
\text { 4/28 } 10 \mathrm{mg} \text { once } \\
\text { daily }\end{array}$ \\
\hline$[10,11]^{\mathrm{c}}$ & 9 & $9 / 9$ & 9/9 acute & 9/9 primary & No & $\begin{array}{l}\text { 4/9 standard } \\
\text { dose } \\
\text { 5/9 } 10 \mathrm{mg} \text { once } \\
\text { daily }\end{array}$ \\
\hline [12] & $9^{d}$ & $4 / 9$ & 9/9 acute & 9/9 secondary & No & $\begin{array}{c}\text { 9/9 20 mg once } \\
\text { daily }\end{array}$ \\
\hline [13] & 3 & $2 / 3$ & $3 / 3$ acute & $3 / 3$ secondary & No & $\begin{array}{c}\text { 3/3 } 15 \text { mg twice } \\
\text { daily }\end{array}$ \\
\hline$[14]$ & 2 & $2 / 2$ & $2 / 2$ acute & $2 / 2$ secondary & $\mathrm{Yes}^{\mathrm{e}}$ & $\begin{array}{l}\text { 2/2 Standard } \\
\text { dose }\end{array}$ \\
\hline [15] & 1 & No & Acute & Secondary & No & Not specified \\
\hline [16] & 1 & Yes & Acute & Secondary & No & Standard dose \\
\hline [17] & 1 & Yes & Acute & Primary & No & $\begin{array}{l}20 \text { mg twice } \\
\text { daily }\end{array}$ \\
\hline [18] & 1 & No & Acute & Primary & No & $10 \mathrm{mg}$ once daily \\
\hline [19] & 1 & Yes & Acute & Primary & No & Standard dose \\
\hline [20] & 1 & Yes & Acute & Secondary & No & Standard dose \\
\hline [21] & 1 & Yes & Acute $^{f}$ & Secondary & No & $20 \mathrm{mg}$ once daily \\
\hline$[22]$ & 1 & No & Acute & Primary & No & $\begin{array}{l}15 \mathrm{mg} \text { twice } \\
\text { daily }\end{array}$ \\
\hline [23] & 1 & Yes & Acute & Secondary & No & Not specified \\
\hline$[24,25]^{\mathrm{g}}$ & 1 & Yes & Acute & Primary & No & Standard dose \\
\hline [26] & 1 & Yes & Subacute & Primary & No & $10 \mathrm{mg}$ once daily \\
\hline [27] & 1 & Yes & Acute & Primary & No & Not specified \\
\hline [28] & 1 & Yes & Acute & Secondary & No & Standard dose \\
\hline [29] & 1 & Yes & Acute & Secondary & No & Not specified \\
\hline Total (\%) & 65 & $\begin{array}{c}40 / 65 \\
(61.5 \%)\end{array}$ & $\begin{array}{c}55 / 65 \text { acute }(84.6 \%) \\
10 / 65 \text { subacute }(15.4 \%)\end{array}$ & $\begin{array}{c}\text { 29/65 primary } \\
(44.6 \%) \\
\text { 36/65 secondary } \\
(55.4 \%)\end{array}$ & $\begin{array}{c}1 / 65 \text { extension of } \\
\text { thrombosis }(1.5 \%) \\
1 / 65 \text { major bleeding } \\
(1.5 \%)\end{array}$ & $\begin{array}{l}33 / 65 \text { standard } \\
\text { dose }(51 \%) \\
28 / 65 \text { other } \\
\text { doses }(43 \%) \\
4 / 65 \text { not } \\
\text { specified }(6 \%)\end{array}$ \\
\hline Our case & 1 & Yes & Subacute & Secondary & No & Standard dose \\
\hline
\end{tabular}

${ }^{*}$ See text for details. ${ }^{a}$ Possible extension of a catheter-related arm DVT with full recovery after catheter removal and despite the continuation of rivaroxaban. ${ }^{\mathrm{b}}$ One rectal bleeding episode with a known gastric cancer occurred 9 days after discontinuing rivaroxaban and while receiving fondaparinux, so not included. ${ }^{c}$ The articles are combined because the 3 patients first reported by Ng et al. are also included among the 9 patients reported by Ong et al. ${ }^{\mathrm{d}}$ Out of the 11 patients who received rivaroxaban, 2 tested negative for HIT antibodies and thus are excluded in this table. ${ }^{\mathrm{e}}$ Moderate hemoptysis secondary to known squamous cell lung cancer. ${ }^{\mathrm{f}}$ Platelets dropped $<50 \%$ but did not drop below $150 \times 10^{9} / \mathrm{L} .{ }^{\mathrm{g}}$ Articles in Spanish but data from Barlow et al.

duration of action, argatroban and bivalirudin are preferred in critically ill patients, patients with high bleeding risk, or patients who may need urgent procedures. However, they are expensive and require a hospital stay for parenteral administration and frequent monitoring. Fondaparinux is being increasingly used in clinically stable patients at average risk of bleeding. Danaparoid is no longer available in the United States and parts of Europe [8]. In our case, the patient's stable condition and the average risk of bleeding is the reason to choose fondaparinux as a nonheparin anticoagulant initially.

Accumulating evidence suggests that DOACs are effective in treating HIT, without stimulating HIT antibodies.
DOACs are an attractive treatment option for HIT as they offer many potential benefits, including ease of administration, rapid onset of action, no required monitoring, the competitive cost to other anticoagulants, and efficacy during longer-term anticoagulation. However, the evidence for the efficacy of DOACs for HIT is only based on prospective cohort studies or retrospective case series while an RCT is lacking [2]. Ideally, an RCT demonstrating the efficacy of a DOAC in HIT is preferred before it becomes an approved treatment. However, the rarity of HIT and the challenges in diagnosis delay any trial recruitment.

With respect to the choice of a DOAC, most of the published experience in HIT is with rivaroxaban which is an 
oral direct factor Xa inhibitor. After carrying out a search on PubMed and MEDLINE database using the words "heparininduced thrombocytopenia," and "rivaroxaban," 59 references were identified, dating from 2008 to 2020. Until February 2020, twenty-one articles in English contained reports of HIT cases treated with rivaroxaban: 1 prospective study, 5 retrospective cohort studies, and 14 case reports, totaling 65 patients. In the previously discussed literature, $61.5 \%$ of cases had HIT-related thrombosis, treatment with rivaroxaban was started while the patient was still thrombocytopenic (i.e., acute HIT) in $84.6 \%$ of cases, while it was initiated after platelet recovery (i.e., subacute HIT) in 15.4\%. Out of the 65 cases, one (1.5\%) had recurrent venous thromboembolism and one case (1.5\%) experienced a moderate bleeding episode while receiving rivaroxaban (Table 1).

Rivaroxaban was the initial nonheparin anticoagulant selected to treat HIT (i.e., primary therapy of HIT) in $44.6 \%$ of HIT cases. Switch from another parenteral nonheparin anticoagulant to rivaroxaban therapy (i.e., secondary therapy of HIT) was noticed in $55.4 \%$ of cases. Half of the published cases used the standard dose regimen for venous thromboembolism: $15 \mathrm{mg}$ twice daily for the initial 21 days followed by $20 \mathrm{mg}$ once daily, while others used different dosing regimens (Table 1 ).

Of all the DOACs, rivaroxaban has the largest body of literature for its use in HIT and is the only DOAC that has been evaluated in a prospective study. While the current experience remains limited, it is suggestive of the potential role of rivaroxaban in HIT, which has led to its integration into the 2018 ASH guidelines with a conditional recommendation [8].

In our case, after the initial use of fondaparinux for 3 days, platelets recovered, and rivaroxaban was started before discharging the patient. We used the standard dose of rivaroxaban: $15 \mathrm{mg}$ twice daily for the initial 21 days followed by $20 \mathrm{mg}$ once daily for 3 months. After 3 months of treatment, rivaroxaban was stopped.

In conclusion, this case report demonstrates the effectiveness and safety of rivaroxaban in the treatment of HIT. Given the rarity of HIT itself, there are no consensus management guidelines for the treatment of HIT with rivaroxaban. More research is needed including encouraging physicians to present outcome data when using rivaroxaban, creating registries of HIT treatment with rivaroxaban and conducting studies that compare rivaroxaban and other nonheparin anticoagulants.

\section{Data Availability}

Access to data is restricted to keep patient's privacy. However, if deemed necessary, data will be provided by the corresponding author upon reasonable request after approval from the needed institutional committee.

\section{Ethical Approval}

This research was approved by the ethics and research committee of Jahra Hospital, Ministry of Health (HOM), Kuwait.

\section{Conflicts of Interest}

The authors declare that they have no conflicts of interest.

\section{References}

[1] G. M. Arepally, "Heparin-induced thrombocytopenia," Blood, vol. 129, no. 21, pp. 2864-2872, 2017.

[2] T. E. Warkentin, M. Pai, and L.-A. Linkins, "Direct oral anticoagulants for treatment of HIT: update of Hamilton experience and literature review," Blood, vol. 130, no. 9, pp. 1104-1113, 2017.

[3] B. H. Chong, "Heparin-induced thrombocytopenia," Journal of Thrombosis and Haemostasis, vol. 1, no. 7, pp. 1471-1478, 2003.

[4] T. Warkentin, C. Hayward, L. Boshkov et al., "Sera from patients with heparin-induced thrombocytopenia generate platelet-derived microparticles with procoagulant activity: an explanation for the thrombotic complications of heparininduced thrombocytopenia," Blood, vol. 84, no. 11, pp. 3691-3699, 1994.

[5] C. Kearon, E. A. Akl, A. J. Comerota et al., "Antithrombotic therapy for VTE disease: antithrombotic therapy and prevention of thrombosis, 9th ed: American College of Chest Physicians evidence-based clinical practice guidelines [published correction appears in chest. 2012 Dec; 142 (6): 1698-1704]," Chest, vol. 141, no. 2, pp. e419S-e496S, 2012.

[6] N. Martel, J. Lee, and P. S. Wells, "Risk for heparin-induced thrombocytopenia with unfractionated and low-molecularweight heparin thromboprophylaxis: a meta-analysis," Blood, vol. 106, no. 8, pp. 2710-2715, 2005.

[7] G. K. Lo, D. Juhl, T. E. Warkentin, C. S. Sigouin, P. Eichler, and A. Greinacher, "Evaluation of pretest clinical score (4 T's) for the diagnosis of heparin-induced thrombocytopenia in two clinical settings," Journal of Thrombosis and Haemostasis, vol. 4, no. 4, pp. 759-765, 2006.

[8] A. Cuker, G. M. Arepally, B. H. Chong et al., "American Society of Hematology 2018 guidelines for management of venous thromboembolism: heparin-induced thrombocytopenia," Blood Advances, vol. 2, no. 22, pp. 3360-3392, 2018.

[9] L. A. Linkins, T. E. Warkentin, M. Pai et al., "Rivaroxaban for treatment of suspected or confirmed heparin-induced thrombocytopenia study," Journal of Thrombosis and Haemostasis, vol. 14, no. 6, pp. 1206-1210, 2016.

[10] S. Y. Ong, Y. A. Chin, H. Than et al., "Rivaroxaban for heparin-induced thrombocytopenia: adding to the evidence," Annals of Hematology, vol. 96, no. 3, pp. 525-527, 2017.

[11] H. J. Ng, H. Than, and E. C. Y. Teo, "First experiences with the use of rivaroxaban in the treatment of heparin-induced thrombocytopenia," Thrombosis Research, vol. 135, no. 1, pp. 205-207, 2015.

[12] M. Sharifi, C. Bay, Z. Vajo, W. Freeman, M. Sharifi, and F. Schwartz, "New oral anticoagulants in the treatment of heparin- induced thrombocytopenia," Thrombosis Research, vol. 135, no. 4, pp. 607-609, 2015.

[13] K. A. Davis and D. O. Davis, "Direct acting oral anticoagulants for the treatment of suspected heparin-induced thrombocytopenia," European Journal of Haematology, vol. 99, no. 4, pp. 332-335, 2017.

[14] P. R. Kunk, J. Brown, M. McShane, S. Palkimas, and B. Gail Macik, "Direct oral anticoagulants in hypercoagulable states," Journal of Thrombosis and Thrombolysis, vol. 43, no. 1, pp. 79-85, 2017. 
[15] I. Kopolovic and T. E. Warkentin, "Progressive thrombocytopenia after cardiac surgery in a 67-year-old man," Canadian Medical Association Journal, vol. 186, no. 12, pp. 929-933, 2014.

[16] P. Hantson, C. Lambert, and C. Hermans, "Rivaroxaban for arterial thrombosis related to heparin-induced thrombocytopenia," Blood Coagulation \& Fibrinolysis, vol. 26, no. 2, pp. 205-206, 2015.

[17] L. Abouchakra, Z. Khabbaz, S. Abouassi, and G. Badaoui, "Rivaroxaban for treatment of heparin-induced thrombocytopenia after cardiac surgery: a case report," The Journal of Thoracic and Cardiovascular Surgery, vol. 150, no. 2, pp. e19-20, 2015

[18] B. Tardy-Poncet, M. Piot, A. Montmartin, A. Burdier, E. Chalayer, and B. Tardy, "Delayed-onset heparin-induced thrombocytopenia without thrombosis in a patient receiving postoperative thromboprophylaxis with rivaroxaban," Thrombosis and Haemostasis, vol. 114, no. 3, pp. 652-654, 2015.

[19] M. Samoš, T. Bolek, J. Ivanková et al., "Heparin-induced thrombocytopenia presenting with deep venous thrombosis and pulmonary embolism successfully treated with rivaroxaban: clinical case report and review of current experiences," Journal of Cardiovascular Pharmacology, vol. 68, no. 5, pp. 391-394, 2016.

[20] J. M. Casan, G. Grigoriadis, N. Chan, and S. Chunilal, "Rivaroxaban in treatment refractory heparin-induced thrombocytopenia," BMJ Case Reports, vol. 2016, 2016.

[21] M. Sartori, E. Favaretto, M. Cini, C. Legnani, and B. Cosmi, "Rivaroxaban in the treatment of heparin-induced thrombocytopenia," Journal of Thrombosis and Thrombolysis, vol. 40, no. 3, pp. 392-394, 2015.

[22] M. Vavlukis, I. Kotlar, H. Taravari, L. Poposka, and S. Kedev, "Can rivaroxaban be a drug of choice for treating heparininduced thrombocytopenia in a patient with pulmonary thromboembolism?" The Anatolian Journal of Cardiology, vol. 18, no. 1, pp. 77-79, 2017.

[23] A. Tvito, T. Bakchoul, J. M. Rowe, A. Greinacher, and C. Ganzel, "Severe and persistent heparin-induced thrombocytopenia despite fondaparinux treatment," American Journal of Hematology, vol. 90, no. 7, pp. 675-678, 2015.

[24] O. G. Rivera, S. O. Corsi, J. A. Pavlovic, S. A. Aizman, and A. P. Varas, "Rivaroxaban para el tratamiento de la trombocitopenia inducida por heparina. Caso clínico," Revista Médica de Chile, vol. 145, no. 9, pp. 1213-1217, 2017.

[25] A. Barlow, B. Barlow, T. Reinaker, and J. Harris, "Potential role of direct oral anticoagulants in the management of heparin-induced thrombocytopenia," Pharmacotherapy: The Journal of Human Pharmacology and Drug Therapy, vol. 39, no. 8, pp. 837-853, 2019.

[26] H. Huang, Y. Lin, R. X. Yao, M. Q. He, and X. J. Lin, "Successful treatment of severe heparin-induced thrombocytopenia with intravenous immunoglobulin, platelet transfusion and rivaroxaban: a case report," Chinese Medical Sciences Journal, vol. 34, no. 1, pp. 60-64, 2019.

[27] M. J. Bienz, P. Obrocki, J. Russell, R. Jena, and I. A. Mendichovszky, "Heparin-induced thrombocytopaenia presenting as acute aortic mural thrombosis," BJR Case Reports, vol. 5, no. 1, 2018.

[28] F. Manji, T. E. Warkentin, J.-A. I. Sheppard, and A. Lee, "Fondaparinux cross-reactivity in heparin-induced thrombocytopenia successfully treated with high-dose intravenous immunoglobulin and rivaroxaban," Platelets, vol. 31, no. 1, pp. 124-127, 2020.
[29] T. Bakchoul, O. Borst, R. Riessen et al., "Autoimmune heparin-induced thrombocytopenia after transcatheter aortic valve implantation: successful treatment with adjunct highdose intravenous immunoglobulin," TH Open, vol. 3, no. 2, pp. e200-e202, 2019. 